\title{
ON GENERATING SUBGROUPS OF THE AFFINE GROUP ON THE PLANE BY PAIRS OF INFINITESIMAL TRANSFORMATIONS*
}

\author{
RICHARD M. KOCH AND FRANKLIN LOWENTHAL
}

\begin{abstract}
Let $G$ be a Lie group with Lie algebra $g$ and let $X$ and $Y$ be elements of $g$. If every element of $G$ can be written as a product of elements taken alternately from $\exp (t X)$ and $\exp (t Y), X$ and $Y$ are said to generate $G$. A classification will be obtained here of all Lie subgroups of the affine group acting on the plane; for each such group, necessary and sufficient conditions will be found that a pair of elements in the Lie algebra generate the group. All but three subgroups of the affine group can be so generated.
\end{abstract}

I. Introduction. The real affine group $A(2)$ acting on the plane is the set of all transformations from $R^{2}$ to $R^{2}$ of the form $v \rightarrow A v+\ell$, where $A \in G L(2, R)$ and $\ell \in R^{2}$. From now on denote such a transformation by $\langle A, \ell\rangle$. Then $\langle A, \ell\rangle \circ\langle B, m\rangle=\langle A B, \ell+A m\rangle$. The Lie algebra $a(2)$ of $A(2)$ consists of all $\langle A, \ell\rangle$ with $A \in M_{2}(R)$ and $\ell \in R^{2} ; \quad[\langle A, \ell\rangle,\langle B, m\rangle]=\langle A B-B A, A m-B \ell\rangle$. We shall determine all Lie subalgebras of $a(2)$ up to conjugacy, and thereby all connected Lie subgroups of $A(2)$ up to conjugacy.

A connected Lie group $G$ is generated by a pair of one-parameter subgroups if every element of $G$ can be written as a finite product of elements chosen alternately from the two one-parameter subgroups. This happens just in case the Lie algebra of $G$ is generated by the corresponding pair of infinitesimal transformations, because the set of all such finite products is an arcwise connected subgroup of $G$ and so a Lie subgroup by Yamabe's theorem [4]. It is known that all connected subgroups of the Moebius group $w=(\alpha z+\beta) /(\nu z+\zeta), \alpha, \beta, \nu$, and $\zeta$ complex, can be generated by an appropriate pair of infinitesimal transformations with the exception of the group $w=\alpha z+\beta, \alpha>0$ [2]. This group is also a subgroup of $A(2)$; we will show that all subgroups of $A(2)$, with the exception of this group and two others, can be generated by a suitable pair of infinitesimal transformations.

*Classification: Primary, 22E15 and 50D10; Secondary, None.

Received by the Editors April 22, 1974.

Copyright $\odot 1976$ Rocky Mountain Mathematics Consortium 


\section{Subalgebras of $M_{2}(R)$.}

Theоrem 1. Let $g$ be a subalgebra of $M_{2}(R)$. Then $g$ is conjugate to precisely one of the following:

(1) $\{0\}$

(3) $R\left(\begin{array}{rr}\lambda & -1 \\ 1 & \lambda\end{array}\right)$ where $0 \leqq \lambda$

(5) $R\left(\begin{array}{ll}1 & 1 \\ 0 & 1\end{array}\right)$

(7) $\left\{\left(\begin{array}{ll}a & b \\ 0 & a\end{array}\right)\right\}$

(9) $\left\{\left(\begin{array}{ll}\lambda a & b \\ 0 & (\lambda+1) a\end{array}\right)\right\}$ where $\lambda \in R$

(11) $s \ell(2, R)$
(2) $R\left(\begin{array}{ll}1 & 0 \\ 0 & \lambda\end{array}\right)$ where $|\lambda| \leqq 1$

(4) $R\left(\begin{array}{ll}0 & 1 \\ 0 & 0\end{array}\right)$

(6) $\left\{\left(\begin{array}{ll}a & 0 \\ 0 & b\end{array}\right)\right\}$

(8) $\left\{\left(\begin{array}{rr}a & -b \\ b & a\end{array}\right)\right\}$

(10) $\left\{\left(\begin{array}{ll}a & b \\ 0 & d\end{array}\right)\right\}$

(12) $M_{2}(R)$.

Proof. The rational canonical form theorem implies that each onedimensional $g$ is conjugate to an algebra listed in (2) through (5).

Lemma. If $g \subset M_{2}(R)$ is isomorphic to $R \oplus R$, g contains the identity matrix $I$.

Proof. Suppose not and let $e$ and $f$ generate $g$. Choose $h \in M_{2}(R)$ so $\{e, f, I, h\}$ is a basis for $M_{2}(R)$. Then $s \ell(2, R)=\left[M_{2}(R), M_{2}(R)\right]$ is generated by $[e, h]$ and $[f, h]$, although it is three dimensional.

Assume that $g$ is isomorphic to $R \oplus R$ and choose $e$ so $e$ and $I$ generate $g$; after suitable conjugation we may suppose that $e$ is one of the matrices listed in (2) through (5); after subtracting a suitable multiple of $I$, we may suppose that $e$ is one of $\left(\begin{array}{ll}1 & 0 \\ 0 & 0\end{array}\right),\left(\begin{array}{cc}0 & -1 \\ 1 & 0\end{array}\right),\left(\begin{array}{ll}0 & 1 \\ 0 & 0\end{array}\right)$. Thus $g$ is conjugate to 6,7 , or 8 ; no two of these algebras are conjugate because every matrix in 6 is diagonalizable and every matrix in 7 has at least one real eigenvalue.

If $g$ is two dimensional and non-abelian, $g$ has a basis $\{e, f\}$ so $[e, f]=e$. Notice that $\operatorname{tr} e=0$; after suitable conjugation, then, $e=\left(\begin{array}{cc}1 & 0 \\ 0 & -1\end{array}\right),\left(\begin{array}{cc}0 & -1 \\ 1 & 0\end{array}\right)$, or $\left(\begin{array}{ll}0 & 1 \\ 0 & 0\end{array}\right)$. Let $f=\left(\begin{array}{ll}a & b \\ c & d\end{array}\right) ;[e, f]$ is then $\left(\begin{array}{rr}0 & 2 b \\ -2 c & 0\end{array}\right)$, $\left(\begin{array}{ccc}-b-c & a-d \\ a-d & b+c\end{array}\right)$, or $\left(\begin{array}{cc}c & d-a \\ 0 & -c\end{array}\right)$; this can equal $e$ only if $e=\left(\begin{array}{ll}0 & 1 \\ 0 & 0\end{array}\right)$ and $f=\left(\begin{array}{ccc}a & b \\ 0 & a+1\end{array}\right)$, so $g$ is conjugate to an algebra listed in (9). Distinct $\lambda$ 's give distinct conjugacy classes of algebras, for if $g$ is conjugate to $\left.\left\{\begin{array}{cc}\lambda a & b \\ 0 & (\lambda+1) a\end{array}\right)\right\}, g$ contains an element with two distinct eigenvalues one unit apart, and these eigenvalues must be $\lambda$ and $\lambda+1$. 
Suppose $\operatorname{dim} g=3$ and $I \notin g$. Then $g \oplus R I=M_{2}(R)$ and $s \ell(2, R)$ $=\left[M_{2}(R), M_{2}(R)\right] \subset[g, g] \subset g$, so $s l(2, R)=g$.

Finally suppose $\operatorname{dim} g=3$ and $I \in g$. Then $s \ell(2, R) \cap g$ is a two dimensional Lie algebra and so conjugate to one of $6,7,8,9$; since any algebra conjugate to $s l(2, R) \cap g$ is contained in $s l(2, R), s l(2, R) \cap g$ is conjugate to $\left(\begin{array}{cc}-a / 2 & b \\ 0 & a / 2\end{array}\right)$ and $g$ is conjugate to 10 .

THEOREM 2. Every connected Lie subgroup of $G L(2, R)$ is conjugate to precisely one of the following:

(1) $\{I\}$

(3) $\left\{e^{\lambda t}\left(\begin{array}{rr}\cos t & -\sin t \\ \sin t & \cos t\end{array}\right)\right\}$ where $0 \leqq \lambda$

(5) $\left\{e^{t}\left(\begin{array}{ll}1 & t \\ 0 & 1\end{array}\right)\right\}$

(7) $\left\{\left(\begin{array}{ll}a & b \\ 0 & a\end{array}\right) \mid a>0\right\}$

(9) $\left\{\left(\begin{array}{ll}a^{\lambda} & b \\ 0 & a^{\lambda+1}\end{array}\right) \mid a>0\right\}$ where $\lambda \in R$
(2) $\left\{\left(\begin{array}{ll}e^{t} & 0 \\ 0 & e^{\lambda t}\end{array}\right)\right\}$ where $|\lambda| \leqq 1$

(4) $\left\{\left(\begin{array}{ll}1 & t \\ 0 & 1\end{array}\right)\right\}$

(6) $\left\{\left(\begin{array}{ll}a & 0 \\ 0 & b\end{array}\right) \mid a>0, b>0\right\}$

(8) $\left\{a\left(\begin{array}{rr}\cos t & -\sin t \\ \sin t & \cos t\end{array}\right) \mid a>0\right\}$

(10) $\left\{\left(\begin{array}{ll}a & b \\ 0 & d\end{array}\right) \mid a>0, d>0\right\}$

(11) $\left\{\left(\begin{array}{ll}a & b \\ c & d\end{array}\right) \mid a d-b c=1\right\}$ (12) $\left\{\left(\begin{array}{ll}a & b \\ c & d\end{array}\right) \mid a d-b c>0\right\}$.

Proof. An immediate consequence of Theorem 1.

III. Subalgebras of $a(2)$. Let $g$ be a subalgebra of $a(2)$; define $g_{0}=$ $\left\{A \in M_{2}(R) \mid\right.$ there exists $\ell \in R^{2}$ such that $\left.\langle A, \ell\rangle \in g\right\}$ and $V=$ $\left\{l \in R^{2} \mid\langle 0, \ell\rangle \in g\right\}$. Then $g_{0}$ is a subalgebra of $M_{2}(R)$ and $V$ is a subspace of $R^{2} ; g_{0}(V) \subset V$ because $[\langle A, \ell\rangle,\langle 0, m\rangle]=\langle 0, A m\rangle$. The following sequence is exact:

$$
0 \rightarrow V \rightarrow g \rightarrow g_{0} \rightarrow 0
$$

Notice that conjugation of $\langle A, \ell\rangle \in a(2)$ by $\langle B, m\rangle \in A(2)$ yields $\left\langle B A B^{-1}, B l-B A B^{-1} m\right\rangle$. In particular $g_{0}$ becomes $B g_{0} B^{-1}$ and $V$ becomes $B V$. 
TheOREM 3. The pair $\left\{g_{0}, V\right\}$ is conjugate to precisely one of the following:

(a) $\left\{g_{0},\{0\}\right\}$ where $g_{0}$ is one of the algebras listed in Theorem 1

(b) $\left\{g_{0}, R^{2}\right\}$ where $g_{0}$ is one of the algebras listed in Theorem 1

(c) $\left\{g_{0}, R\left(\begin{array}{l}1 \\ 0\end{array}\right)\right\}$ where $g_{0}$ is one of $\{0\}, R\left(\begin{array}{ll}1 & 0 \\ 0 & 1\end{array}\right), R\left(\begin{array}{ll}1 & 0 \\ 0 & -1\end{array}\right), R\left(\begin{array}{ll}0 & 1 \\ 0 & 0\end{array}\right)$, $\left.\boldsymbol{R}\left(\begin{array}{ll}1 & 1 \\ 0 & 1\end{array}\right),\left\{\begin{array}{ll}a & 0 \\ 0 & b\end{array}\right)\right\},\left\{\left(\begin{array}{cc}\lambda a & b \\ 0 & (\lambda+1) a\end{array}\right)\right\},\left\{\left(\begin{array}{ll}a & b \\ 0 & a\end{array}\right)\right\},\left\{\left(\begin{array}{ll}a & b \\ 0 & d\end{array}\right)\right\}$

(d) $\left\{\boldsymbol{R}\left(\begin{array}{ll}1 & 0 \\ 0 & \lambda\end{array}\right), \boldsymbol{R}\left(\begin{array}{l}1 \\ 0\end{array}\right)\right\}$ or $\left\{\boldsymbol{R}\left(\begin{array}{ll}1 & 0 \\ 0 & \lambda\end{array}\right), \boldsymbol{R}\left(\begin{array}{l}0 \\ 1\end{array}\right)\right\}$ for $|\lambda|<1$.

Proof. We can assume that $g_{0}$ is one of the algebras listed in Theorem 1. The condition $g_{0}(V) \subset V$ puts no restriction on $V$ if $\operatorname{dim} V$

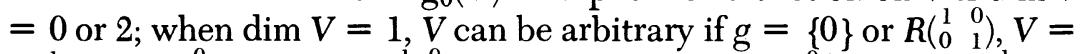
$\boldsymbol{R}\left(\begin{array}{l}1 \\ 0\end{array}\right)$ or $R\left(\begin{array}{l}0 \\ 1\end{array}\right)$ if $g=\boldsymbol{R}\left(\begin{array}{ll}1 & 0 \\ 0 & \lambda\end{array}\right)-1 \leqq \lambda<1$ or $\left\{\left(\begin{array}{ll}a & 0 \\ 0 & b\end{array}\right)\right\}, V=\boldsymbol{R}\left(\begin{array}{l}1 \\ 0\end{array}\right)$ if $\left.g=\boldsymbol{R}\left(\begin{array}{ll}0 & 1 \\ 0 & 0\end{array}\right), \quad \boldsymbol{R}\left(\begin{array}{ll}1 & 1 \\ 0 & 1\end{array}\right), \quad\left\{\left(\begin{array}{cc}\lambda a & b \\ 0 & (\lambda+1) b\end{array}\right)\right\}, \quad\left\{\begin{array}{ll}a & b \\ 0 & a\end{array}\right)\right\}, \quad$ or $\left\{\left(\begin{array}{ll}a & b \\ 0 & d\end{array}\right)\right\}$, and other-

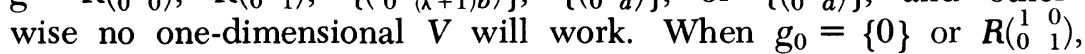
$B g_{0} B^{-1}=g_{0}$ for all $B$; applying an appropriate $B$ to $V$ we can assume $V=R\left(\begin{array}{l}1 \\ 0\end{array}\right)$ If $g_{0}=\left\{\left(\begin{array}{ll}a & 0 \\ 0 & b\end{array}\right)\right\} \quad$ or $R\left(\begin{array}{ll}1 & 0 \\ 0 & -1\end{array}\right), \quad\left(\begin{array}{ll}0 & 1 \\ 1 & 0\end{array}\right) g_{0}\left(\begin{array}{ll}0 & 1 \\ 1 & 0\end{array}\right)^{-1}=g_{0} \quad$ and $\left(\begin{array}{ll}0 & 1 \\ 1 & 0\end{array}\right) R\left(\begin{array}{l}1 \\ 0\end{array}\right)=\boldsymbol{R}\left(\begin{array}{l}0 \\ 1\end{array}\right)$; thus $V$ can be taken to be $\boldsymbol{R}\left(\begin{array}{l}1 \\ 0\end{array}\right)$. If $g_{0}=\boldsymbol{R}\left(\begin{array}{ll}1 & 0 \\ 0 & \lambda\end{array}\right)$ for $|\lambda|<1$ and $B g_{0} B^{-1}=g_{0}, B=\left(\begin{array}{ll}a & 0 \\ 0 & b\end{array}\right)$ so $R\left(\begin{array}{l}1 \\ 0\end{array}\right)$ and $R\left(\begin{array}{l}0 \\ 1\end{array}\right)$ are not conjugate.

Choose a subspace $V_{1}$ of $R^{2}$ so $V \oplus V_{1}=R^{2}$. Whenever $A \in g_{0}$, there is a unique $\varphi(A) \in V_{1}$ so $\langle A, \varphi(A)\rangle \in g$. Clearly $g=\{\langle A, \varphi(A)$ $\left.+\ell\rangle \mid A \in g_{0}, \ell \in V\right\}$.

Notice that $\varphi: g_{0} \rightarrow V_{1}$ is linear. Let $P: R^{2} \rightarrow V_{1}$ be the obvious projection map. Then $\varphi([A, B])=P\{A \varphi(B)-B \varphi(A)\}$ since $[\langle A, \varphi(A)\rangle,\langle B, \varphi(B)\rangle]=\langle[A, B], A \varphi(B)-B \varphi(A)\rangle$.

Conjugation of $g$ by $\langle I, m\rangle$ leaves $g_{0}$ and $V$ fixed and converts $\langle A, \varphi(A)\rangle$ to $\langle A, \varphi(A)-A m\rangle$. Hence we may replace $\varphi(A)$ by $P\{\varphi(A)-A m\}$ and obtain a conjugate algebra.

If $g_{0}=\{0\}, \varphi=0$. If $g_{0}=R e$ where $e=\left(\begin{array}{ll}1 & 0 \\ 0 & \lambda\end{array}\right) 0<|\lambda| \leqq 1,\left(\begin{array}{cc}\lambda & -1 \\ 1 & \lambda\end{array}\right)$ $0 \leqq \lambda$ or $\left(\begin{array}{ll}1 & 1 \\ 0 & 1\end{array}\right), m$ can be found so $\varphi(e)=e m$ since $\operatorname{det} e \neq 0$. Thus after conjugation $\varphi=0$. If $I \in g_{0}$ we can suppose $\varphi(I)=0$ since $I$ is nonsingular; whenever $A \in g_{0}, 0=\varphi([I, A])=P\{\varphi(A)\}=\varphi(A)$, so $\varphi=0$. If $g_{0}=s l(2, R), \varphi=0$ unless $V=\{0\}$ and $V_{1}=R^{2}$. In this case let $e=\left(\begin{array}{cc}1 & 0 \\ 0 & -1\end{array}\right), f=\left(\begin{array}{ll}0 & 1 \\ 0 & 0\end{array}\right), \quad g=\left(\begin{array}{ll}0 & 0 \\ 1 & 0\end{array}\right)$; as usual we may assume $\varphi(e)=0$. Then $2 \varphi(f)=\varphi[e, f]=\left(\begin{array}{ll}1 & 0 \\ 0 & 0\end{array}\right) \varphi(f)$, so $\varphi(f)=0$. Similarly $-2 \varphi(g)=\varphi[e, g]=\left(\begin{array}{cc}1 & 0 \\ 0 & -1\end{array}\right) \varphi(g)$, so $\varphi(g)=0$ and $\varphi=0$. If $g_{0}=\left\{\left(\begin{array}{cc}\lambda a & b \\ 0 & (\lambda+1) a\end{array}\right)\right\}$, let $e=\left(\begin{array}{cc}\lambda & 0 \\ 0 & \lambda+1\end{array}\right), f=\left(\begin{array}{ll}0 & 1 \\ 0 & 0\end{array}\right)$. Suppose $\lambda \neq 0,-1$, or -2 . Then $\operatorname{det}\left(\begin{array}{cc}\lambda & 0 \\ 0 & \lambda+1\end{array}\right) \neq 0$ and we can suppose $\varphi(e)=0 ; \quad-\varphi(f)=\varphi[e, f]=P\left\{\left(\begin{array}{cc}\lambda & 0 \\ 0 & \lambda+1\end{array}\right) \varphi(f)\right\} . \quad$ If $\quad V=\{0\} \quad$ and

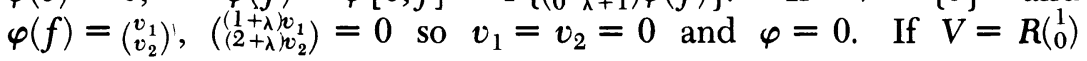


let $V_{1}=R\left(\begin{array}{l}0 \\ 1\end{array}\right)$ and $\varphi(f)=\left(\begin{array}{l}0 \\ v\end{array}\right)$; then $-\left(\begin{array}{l}0 \\ v\end{array}\right)=\left(\begin{array}{c}0 \\ (\lambda+1) v\end{array}\right)$ and $v=0$, so $\varphi=0$. In short, $\varphi$ can be taken to be zero unless $g_{0}=R\left(\begin{array}{ll}0 & 0 \\ 1 & 0\end{array}\right), R\left(\begin{array}{ll}0 & 1 \\ 0 & 0\end{array}\right)$, $\left\{\left(\begin{array}{ll}0 & b \\ 0 & a\end{array}\right)\right\},\left\{\left(\begin{array}{ll}a & b \\ 0 & 0\end{array}\right)\right\}$, or $\left\{\left(\begin{array}{cc}2 a & b \\ 0 & a\end{array}\right)\right\}$. The following five lemmas complete the classification.

LeMma 1. Let $g_{0}=R\left(\begin{array}{ll}1 & 0 \\ 0 & 0\end{array}\right)$. If $V=R\left(\begin{array}{l}0 \\ 1\end{array}\right)$ or $R^{2}, \varphi$ can be taken to be zero. If $V=\{0\}$ or $R\left(\begin{array}{l}1 \\ 0\end{array}\right), \varphi\left(\begin{array}{ll}1 & 0 \\ 0 & 0\end{array}\right)$ can be taken to be 0 or $\left(\begin{array}{l}0 \\ 1\end{array}\right)$.

Proof. If $B g_{0} B^{-1}=g_{0}, B=\left(\begin{array}{ll}a & 0 \\ 0 & d\end{array}\right)$; such a $B$ preserves all possible $V$. Conjugation of $\left\langle\left(\begin{array}{ll}1 & 0 \\ 0 & 0\end{array}\right), \varphi\left(\begin{array}{ll}1 & 0 \\ 0 & 0\end{array}\right)\right\rangle$ by $\left\langle\left(\begin{array}{ll}a & 0 \\ 0 & d\end{array}\right), m\right\rangle$ yields $\left\langle\left(\begin{array}{ll}1 & 0 \\ 0 & 0\end{array}\right)\right.$, $\left.\left(\begin{array}{ll}a & 0 \\ d & d\end{array}\right) \varphi\left(\begin{array}{ll}d & 0 \\ 0\end{array}\right)-\left(\begin{array}{cc}1 & 0 \\ 0\end{array}\right) m\right\rangle$. Clearly $a, d$, and $m$ can be chosen to make the second part of this expression equal 0 or $\left(\begin{array}{l}0 \\ 1\end{array}\right)$. We are interested in the projection of this term on $V_{1}$; since $V_{1}=R^{2}, R\left(\begin{array}{l}0 \\ 1\end{array}\right), R\left(\begin{array}{l}1 \\ 0\end{array}\right)$, and $\{0\}$ when $V=\{0\}, R\left(\begin{array}{l}1 \\ 0\end{array}\right), R\left(\begin{array}{l}0 \\ 1\end{array}\right)$, and $R^{2}$, the lemma follows.

LEMMA 2. Let $g_{0}=R\left(\begin{array}{ll}0 & 1 \\ 0 & 0\end{array}\right)$. If $V=R^{2}, \varphi=0$. If $V=\{0\}$ or $R\left(\begin{array}{l}1 \\ 0\end{array}\right), \varphi\left(\begin{array}{ll}0 & 1 \\ 0 & 0\end{array}\right)$ can be taken to be 0 or $\left(\begin{array}{l}0 \\ 1\end{array}\right)$.

Proof. If $B g_{0} B^{-1}=g_{0}, B=\left(\begin{array}{ll}a & b \\ 0 & d\end{array}\right)$; such a $B$ preserves all possible $V$. Conjugation of $\left\langle\left(\begin{array}{ll}0 & 1 \\ 0 & 0\end{array}\right), \varphi\left(\begin{array}{ll}0 & 1 \\ 0 & 0\end{array}\right)\right\rangle$ by $\left\langle\left(\begin{array}{ll}a & b \\ 0 & d\end{array}\right), m\right\rangle$ yields $\left\langle\left(\begin{array}{ll}0 & a / d \\ 0 & 0\end{array}\right)\right.$, $\left.\left(\begin{array}{ll}a & b \\ 0 & d\end{array}\right) \varphi\left(\begin{array}{ll}0 & 1 \\ 0 & 0\end{array}\right)-\left(\begin{array}{cc}0 & a / d \\ 0 & 0\end{array}\right) m\right\rangle$. Clearly $a=d$ and $m$ can be chosen to make the second part of this expression equal 0 or $\left(\begin{array}{l}0 \\ 1\end{array}\right)$.

LeMmA 3. Let $g_{0}=\left\{\left(\begin{array}{ll}0 & b \\ 0\end{array}\right)\right\}$. If $V=R\left(\begin{array}{l}1 \\ 0\end{array}\right)$ or $R^{2}, \varphi$ can be taken to be zero. If $V=\{0\}, \varphi\left(\begin{array}{cc}0 & b \\ 0 & a\end{array}\right)$ can be taken to be 0 or $\left(\begin{array}{l}a \\ 0\end{array}\right)$.

ProOF. Let $e=\left(\begin{array}{ll}0 & 1 \\ 0 & 0\end{array}\right), \quad f=\left(\begin{array}{ll}0 & 0 \\ 0 & 1\end{array}\right), \quad \varphi(e)=\left(\begin{array}{l}v_{1} \\ v_{2}\end{array}\right), \quad \varphi(f)=\left(\begin{array}{l}w_{1} \\ w_{2}\end{array}\right)$. If $V=\{0\}, \quad\left(\begin{array}{l}v_{1} \\ v_{2}\end{array}\right)=\varphi(e)=\varphi[e, f]=\left(\begin{array}{ll}0 & 1 \\ 0 & 0\end{array}\right) \quad\left(\begin{array}{l}w_{1} \\ w_{2}\end{array}\right)-\left(\begin{array}{ll}0 & 0 \\ 0 & 1\end{array}\right) \quad\left(\begin{array}{l}v_{1} \\ v_{2}\end{array}\right)=$ $\left(\begin{array}{c}w_{2} \\ -v_{2}\end{array}\right)$, so $v_{1}=w_{2}$ and $v_{2}=0$. If $V=R\left(\begin{array}{l}1 \\ 0\end{array}\right)$, let $V_{1}=R\left(\begin{array}{l}0 \\ 1\end{array}\right)$; then $v_{1}=$ $w_{1}=0 \quad$ and $\quad\left(\begin{array}{c}0 \\ -v_{2}\end{array}\right)=\varphi(e)=\varphi[e, f]=P\left\{\left(\begin{array}{c}w_{2} \\ -v_{2}\end{array}\right)\right\}=\left(\begin{array}{c}0 \\ -v_{2}\end{array}\right)$, so $v_{2}$ $=0$.

If $B g_{0} B^{-1}=g_{0}, B=\left(\begin{array}{ll}a & b \\ 0 & d\end{array}\right)$; such a $B$ preserves all possible $V$. Conjugation of $\langle e, \varphi(e)\rangle$ and $\langle f, \varphi(f)\rangle$ by $\left\langle\left(\begin{array}{ll}a & b \\ 0 & d\end{array}\right), m\right\rangle$ yields $\left\langle\left(\begin{array}{ll}0 & \text { ald } \\ 0 & 0\end{array}\right), \quad\left(\begin{array}{ll}a & b \\ 0 & d\end{array}\right) \varphi(e)-\left(\begin{array}{cc}0 & \text { ald } \\ 0 & 0\end{array}\right) m\right\rangle \quad$ and $\left\langle\left(\begin{array}{cc}0 & b / d \\ 0 & 0\end{array}\right), \quad\left(\begin{array}{ll}a & b \\ 0 & d\end{array}\right) \varphi(f)-\right.$ $\left.\left(\begin{array}{lll}0 & b / d \\ 0 & l\end{array}\right) m\right\rangle$. If $V=R\left(\begin{array}{l}1 \\ 0\end{array}\right)$, we are only interested in the projection of the second parts of these expressions on $R\left(\begin{array}{l}0 \\ 1\end{array}\right)$; since $\varphi(e)=0$, the projection of $\left(\begin{array}{ll}a & b \\ 0 & d\end{array}\right) \varphi(e)-\left(\begin{array}{cc}0 & a / d \\ 0 & 0\end{array}\right) m$ is automatically zero; clearly $a=d$, $b=0$, and $m$ can be chosen to make the projection of $\left(\begin{array}{ll}a & b \\ 0 & d\end{array}\right) \varphi(f)$ $-\left(\begin{array}{cc}0 & b / d \\ 0 & 1\end{array}\right) m$ vanish. If $V=\{0\}$, let $\tilde{\varphi}$ be the conjugate of $\varphi$;

$$
\begin{aligned}
\tilde{\varphi}(e)=\frac{d}{a} \tilde{\varphi}\left(\begin{array}{cc}
0 & a / d \\
0 & 0
\end{array}\right) & =\frac{d}{a}\left\{\left(\begin{array}{c}
a b \\
0 d
\end{array}\right)\left(\begin{array}{c}
v_{1} \\
0
\end{array}\right)\right. \\
& \left.-\left(\begin{array}{cc}
0 & a / d \\
0 & 0
\end{array}\right)\left(\begin{array}{c}
m_{1} \\
m_{2}
\end{array}\right)\right\}=\left(\begin{array}{c}
d v_{1}-m_{2} \\
0
\end{array}\right),
\end{aligned}
$$




$$
\begin{aligned}
\tilde{\varphi}(f)= & \tilde{\varphi}\left(\begin{array}{rr}
0 & b / d \\
0 & 1
\end{array}\right)-\frac{b}{d} \tilde{\varphi}(e)=\left(\begin{array}{ll}
a & b \\
0 & d
\end{array}\right)\left(\begin{array}{l}
w_{1} \\
v_{1}
\end{array}\right) \\
& -\left(\begin{array}{rr}
0 & b / d \\
0 & 1
\end{array}\right)\left(\begin{array}{l}
m_{1} \\
m_{2}
\end{array}\right)-\left(\begin{array}{cc}
b v_{1}-b / d m_{2} \\
0
\end{array}\right)=\left(\begin{array}{c}
a w_{1} \\
d v_{1}-m_{2}
\end{array}\right) .
\end{aligned}
$$

Clearly $a, d$, and $m_{2}$ can be chosen to make $\tilde{\varphi}(e)=0$ and $\tilde{\varphi}(f)$ either 0 or $\left(\begin{array}{l}1 \\ 0\end{array}\right)$.

LEMMA 4. Let $g_{0}=\left\{\left(\begin{array}{ll}a & b \\ 0 & 0\end{array}\right)\right\}$. If $V=\{0\}$ or $R^{2}, \varphi$ can be taken to be zero. If $V=R\left(\begin{array}{l}1 \\ 0\end{array}\right), \varphi\left(\begin{array}{ll}a & b \\ 0 & 0\end{array}\right)$ can be taken to be 0 or $\left(\begin{array}{l}0 \\ a\end{array}\right)$.

Proof. Let $e=\left(\begin{array}{ll}0 & 1 \\ 0 & 0\end{array}\right), \quad f=\left(\begin{array}{ll}1 & 0 \\ 0 & 0\end{array}\right), \quad \varphi(e)=\left(\begin{array}{l}v_{1} \\ v_{2}\end{array}\right), \quad \varphi(f)=\left(\begin{array}{l}w_{1} \\ w_{2}\end{array}\right)$ If $V=\{0\}, \quad-\left(\begin{array}{l}v_{1} \\ v_{2}\end{array}\right)=-\varphi(e)=\varphi[e, f]=\left(\begin{array}{ll}0 & 1 \\ 0 & 0\end{array}\right)\left(\begin{array}{l}w_{1} \\ w_{2}\end{array}\right)-\left(\begin{array}{ll}1 & 0 \\ 0 & 0\end{array}\right) \quad\left(\begin{array}{l}w_{2} \\ v_{2}\end{array}\right)=$ $\left(w_{2}-v_{1}\right)$, so $w_{2}=v_{2}=0$. Conjugation by $\left\langle I,\left(\begin{array}{c}w_{1} \\ v_{1}\end{array}\right)\right\rangle$ converts $\langle e, \varphi(e)\rangle$ and $\langle f, \varphi(f)\rangle$ to $\langle e, 0\rangle$ and $\langle f, 0\rangle$, so $\varphi$ can be taken to be zero.

If $V=\boldsymbol{R}\left(\begin{array}{l}1 \\ 0\end{array}\right)$, let $V_{1}=\boldsymbol{R}\left(\begin{array}{l}0 \\ 1\end{array}\right)$; then $v_{1}=w_{1}=0$ and $-\left(\begin{array}{l}0 \\ v_{2}\end{array}\right)=\varphi[e, f]$ $=P\left\{\left(\begin{array}{c}w_{2} \\ 0^{2}\end{array}\right)\right\}=0$, so $v_{2}=0$. If $B g_{0} B^{-1}=g_{0}, B=\left(\begin{array}{ll}a & b \\ 0 & d\end{array}\right)$. Conjugation of $\langle e, 0\rangle$ and $\langle f, \varphi(f)\rangle$ by $\left\langle\left(\begin{array}{ll}a & b \\ 0 & d\end{array}\right),\left(\begin{array}{c}m_{1} \\ m_{2}\end{array}\right)\right\rangle$ yields $\left\langle\left(\begin{array}{cc}0 & a / d \\ 0 & 0\end{array}\right),-\left(\begin{array}{cc}0 & a / d \\ 0 & 0\end{array}\right)\left(\begin{array}{l}m_{1} \\ m_{2}\end{array}\right)\right\rangle$ and $\left\langle\left(\begin{array}{cc}1 & -b / a \\ 0 & 0\end{array}\right)\left(\begin{array}{ll}a & b \\ 0 & d\end{array}\right)\left(\begin{array}{c}0 \\ w_{2}\end{array}\right)-\left(\begin{array}{cc}1 & -b d \\ 0 & 0\end{array}\right)\left(\begin{array}{l}m_{1} \\ m_{2}\end{array}\right)\right\rangle$; we are only interested in the projection of the second parts of these expressions on $R\left(\begin{array}{l}0 \\ 1\end{array}\right)$, so $\tilde{\varphi}(e)=0, \tilde{\varphi}(f)=\left(\begin{array}{c}0 \\ d w_{2}\end{array}\right)$. Clearly $d$ can be chosen so $\tilde{\varphi}(f)=0$ or $\left(\begin{array}{l}0 \\ 1\end{array}\right)$.

LEMMA 5. Let $\left.g_{0}=\left\{\begin{array}{rr}2 a & b \\ 0 & a\end{array}\right)\right\}$. If $V=R^{2}, \varphi$ is zero. If $V=\{0\}$ or $R\left(\begin{array}{l}1 \\ 0\end{array}\right), \varphi\left(\begin{array}{ll}2 a & b \\ 0 & a\end{array}\right)$ can be taken to be 0 or $\left(\begin{array}{l}0 \\ b\end{array}\right)$.

Proof. Let $e=\left(\begin{array}{ll}0 & 1 \\ 0 & 0\end{array}\right), f=\left(\begin{array}{ll}2 & 0 \\ 0 & 1\end{array}\right)$. Since $f$ is non-singular, $\varphi(f)$ can be taken to be zero; let $\varphi(e)=\left(\begin{array}{l}v_{1} \\ v_{2}\end{array}\right)$. If $\mathrm{V}=\{0\},-\left(\begin{array}{l}v_{1} \\ v_{2}\end{array}\right)=-\varphi(e)=$ $\varphi[e, f]=-\left(\begin{array}{ll}2 & 0 \\ 0 & 1\end{array}\right)\left(\begin{array}{l}v_{1} \\ v_{2}\end{array}\right)=\left(\begin{array}{c}-2 v_{1} \\ -v_{2}\end{array}\right)$, so $v_{1}=0$. If $V=R\left(\begin{array}{l}1 \\ 0\end{array}\right)$, let $V_{1}=$ $R\left(\begin{array}{l}0 \\ 1\end{array}\right)$; then $v_{1}=0$.

If $B g_{0} B^{-1}=g_{0}, B=\left(\begin{array}{ll}a & b \\ 0 & d\end{array}\right)$. Conjugation of $\langle e, \varphi(e)\rangle$ and $\langle f, 0\rangle$ by $\left\langle\left(\begin{array}{ll}a & b \\ 0 & d\end{array}\right),\left(\begin{array}{l}m_{1} \\ m_{2}\end{array}\right)\right\rangle$ yields respectively

$$
\begin{gathered}
\left\langle\left(\begin{array}{rr}
0 & a / d \\
0 & 0
\end{array}\right),\left(\begin{array}{ll}
a & b \\
0 & d
\end{array}\right)\left(\begin{array}{c}
0 \\
v_{2}
\end{array}\right)-\left(\begin{array}{rr}
0 & a / d \\
0 & 0
\end{array}\right)\left(\begin{array}{l}
m_{1} \\
m_{2}
\end{array}\right)\right\rangle, \\
\left\langle\left(\begin{array}{rr}
2 & -b / d \\
0 & 1
\end{array}\right),-\left(\begin{array}{rr}
2 & -b / d \\
0 & 1
\end{array}\right)\left(\begin{array}{l}
m_{1} \\
m_{2}
\end{array}\right)\right\rangle .
\end{gathered}
$$

If $V=\{0\}$,

$$
\tilde{\varphi}(e)=\left(\begin{array}{l}
\frac{b d}{a} v_{2}-m_{2} \\
\frac{d^{2}}{a} v_{2}
\end{array}\right) \quad, \quad \tilde{\varphi}(f)=\left(\begin{array}{l}
-2 m_{1}+\frac{b^{2}}{a} v_{2} \\
\frac{b d}{a} v_{2}-m_{2}
\end{array}\right) .
$$


Clearly $a, b, d, m_{1}$, and $m_{2}$ can be chosen so $\tilde{\varphi}(e)=0$ or $\left(\begin{array}{l}0 \\ 1\end{array}\right)$ and $\tilde{\varphi}(f)$ $=0$. If $V=R\left(\begin{array}{l}1 \\ 0\end{array}\right)$, we are only interested in the projections of the

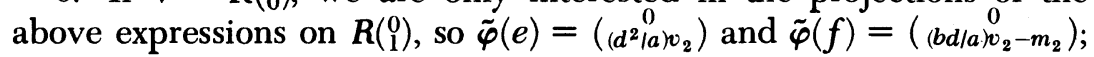
clearly $a, b, d$, and $m_{2}$ can be chosen so $\tilde{\varphi}(e)=0$ or $\left(\begin{array}{l}0 \\ 1\end{array}\right)$ and $\tilde{\varphi}(f)=0$.

Combining the above results, we obtain:

TheOREM 4. Let $g$ be a subalgebra of $a(2)$. Then $g$ is conjugate to precisely one of the following:

A. $\left\{\langle A, 0\rangle \mid A \in g_{0}\right\}$ or $\left\{\langle A, l\rangle \mid A \in g_{0}, \ell \in R^{2}\right\}$ where $g_{0}$ is one of the following

1. $\{0\}$

3. $R\left(\begin{array}{rr}\lambda & -1 \\ 1 & \lambda\end{array}\right)$ where $0 \leqq \lambda$

5. $R\left(\begin{array}{ll}1 & 1 \\ 0 & 1\end{array}\right)$

7. $\left\{\left(\begin{array}{ll}a & b \\ 0 & a\end{array}\right)\right\}$

9. $\left\{\left(\begin{array}{ll}\lambda a & b \\ 0 & (\lambda+1) a\end{array}\right)\right\}$ where $\lambda \in R$

11. $\operatorname{si}(2, R)$
2. $R\left(\begin{array}{cc}1 & 0 \\ 0 & \lambda\end{array}\right)$ where $|\lambda| \leqq 1$

4. $R\left(\begin{array}{ll}0 & 1 \\ 0 & 0\end{array}\right)$

6. $\left\{\left(\begin{array}{ll}a & 0 \\ 0 & b\end{array}\right)\right\}$

8. $\left\{\left(\begin{array}{rr}a & -b \\ b & a\end{array}\right)\right\}$

10. $\left\{\left(\begin{array}{ll}a & b \\ 0 & d\end{array}\right)\right\}$

12. $M_{2}(R)$.

B. $\left\{\langle A, \ell\rangle \mid A \in g_{0}, l \in V\right\}$ where $\left\{g_{0}, V\right\}$ is one of

13. $0, R\left(\begin{array}{l}1 \\ 0\end{array}\right)$

15. $R\left(\begin{array}{ll}1 & 0 \\ 0 & \lambda\end{array}\right), \quad R\left(\begin{array}{l}0 \\ 1\end{array}\right)$

$$
\text { where }|\lambda|<1
$$

17. $R\left(\begin{array}{ll}1 & 1 \\ 0 & 1\end{array}\right), \quad R\left(\begin{array}{l}1 \\ 0\end{array}\right)$

19. $\left\{\left(\begin{array}{ll}a & b \\ 0 & a\end{array}\right)\right\}, R\left(\begin{array}{l}1 \\ 0\end{array}\right)$

21. $\left\{\left(\begin{array}{ll}a & b \\ 0 & d\end{array}\right)\right\}, R\left(\begin{array}{l}1 \\ 0\end{array}\right)$
14. $R\left(\begin{array}{ll}1 & 0 \\ 0 & \lambda\end{array}\right), \quad R\left(\begin{array}{l}1 \\ 0\end{array}\right)$

where $|\lambda| \leqq 1$

16. $R\left(\begin{array}{ll}0 & 1 \\ 0 & 0\end{array}\right), \quad R\left(\begin{array}{l}1 \\ 0\end{array}\right)$

18. $\left\{\left(\begin{array}{ll}a & 0 \\ 0 & b\end{array}\right)\right\}, R\left(\begin{array}{l}1 \\ 0\end{array}\right)$

20. $\left\{\left(\begin{array}{ll}\lambda a & b \\ 0 & (\lambda+1) a\end{array}\right)\right\}, R\left(\begin{array}{l}1 \\ 0\end{array}\right)$

where $\lambda \in R$ 
126

R. M. KOCH AND F. LOWENTHAL

C.

22. $\left\{\left\langle\left(\begin{array}{ll}r & 0 \\ 0 & 0\end{array}\right),\left(\begin{array}{l}0 \\ r\end{array}\right)\right\rangle \mid r \in R\right\}$

23. $\left\{\left\langle\left(\begin{array}{ll}0 & r \\ 0 & 0\end{array}\right),\left(\begin{array}{l}0 \\ r\end{array}\right)\right\rangle \mid r \in R\right\}$

24. $\left\{\left\langle\left(\begin{array}{ll}0 & r \\ 0 & s\end{array}\right),\left(\begin{array}{l}s \\ 0\end{array}\right)\right\rangle \mid r, s \in R\right\}$

25. $\left\{\left\langle\left(\begin{array}{ll}2 r & s \\ 0 & r\end{array}\right),\left(\begin{array}{l}0 \\ s\end{array}\right)\right\rangle \mid r, s \in R\right\}$

26. $\left\{\left\langle\left(\begin{array}{ll}r & 0 \\ 0 & 0\end{array}\right),\left(\begin{array}{l}s \\ r\end{array}\right)\right\rangle \mid r, s \in R\right\}$

27. $\left\{\left\langle\left(\begin{array}{ll}0 & r \\ 0 & 0\end{array}\right),\left(\begin{array}{l}s \\ r\end{array}\right)\right\rangle \mid r, s \in R\right\}$

28. $\left\{\left\langle\left(\begin{array}{cc}r & s \\ 0 & 0\end{array}\right),\left(\begin{array}{c}t \\ r\end{array}\right)\right\rangle \mid r, s, t \in R\right\}$

29. $\left\{\left\langle\left(\begin{array}{cc}2 r & s \\ 0 & r\end{array}\right),\left(\begin{array}{l}t \\ s\end{array}\right)\right\rangle \mid r, s, t \in R\right\}$

TheOREM 5. Let $G$ be a connected Lie subgroup of $A(2)$. Then $G$ is conjugate to precisely one of the following:

A. $\left\{\langle A, 0\rangle \mid A \in G_{0}\right\}$ or $\left\{\langle A, l\rangle \mid A \in G_{0}, \ell \in R^{2}\right\}$ where $G_{0}$ is one of the following,

1. $\{I\}$

3. $\left\{\mathrm{e}^{\lambda t}\left(\begin{array}{rr}\cos t & -\sin t \\ \sin t & \cos t\end{array}\right)\right\}$ where $\lambda \geqq 0$

5. $\left\{\mathrm{e}^{t}\left(\begin{array}{ll}1 & t \\ 0 & 1\end{array}\right)\right\}$

7. $\left\{\left(\begin{array}{ll}a & b \\ 0 & a\end{array}\right) \mid a>0\right\}$

9. $\left\{\left(\begin{array}{ll}a^{\lambda} & b \\ 0 & a^{\lambda+1}\end{array}\right) \mid a>0\right\}$ where $\lambda \in R$

11. $\left\{\left(\begin{array}{ll}a & b \\ c & d\end{array}\right) \quad \mid a d-b c=1\right\}$
2. $\left\{\left(\begin{array}{ll}\mathrm{e}^{t} & 0 \\ 0 & \mathrm{e}^{\lambda t}\end{array}\right)\right\}$ where $|\lambda| \leqq 1$

4. $\left\{\left(\begin{array}{ll}1 & t \\ 0 & 1\end{array}\right)\right\}$

6. $\left\{\left(\begin{array}{ll}a & 0 \\ 0 & b\end{array}\right) \quad \mid a>0, b>0\right\}$

8. $\left\{a\left(\begin{array}{rr}\cos t & -\sin t \\ \sin t & \cos t\end{array}\right) \mid a>0\right\}$

10. $\left\{\left(\begin{array}{ll}a & b \\ 0 & d\end{array}\right) \mid a>0, d>0\right\}$

12. $\left\{\left(\begin{array}{ll}a & b \\ c & d\end{array}\right) \quad \mid a d-b c \neq 0\right\}$ 
B. $\left\{\langle A, l\rangle \mid A \in G_{0}, \ell \in V\right\}$ where $\left\{G_{0}, V\right\}$ is one of

13. $\{I\}, R\left(\begin{array}{l}1 \\ 0\end{array}\right) \quad$ 14. $\left\{\left(\begin{array}{ll}\mathrm{e}^{t} & 0 \\ 0 & \mathrm{e}^{\lambda t}\end{array}\right)\right\}, R\left(\begin{array}{l}1 \\ 0\end{array}\right)$ where $|\lambda| \leqq 1$

15. $\left\{\left(\begin{array}{ll}\mathrm{e}^{t} & 0 \\ 0 & \mathrm{e}^{\lambda t}\end{array}\right)\right\}, R\left(\begin{array}{l}0 \\ 1\end{array}\right) \quad$ 16. $\left\{\left(\begin{array}{ll}1 & t \\ 0 & 1\end{array}\right)\right\}, R\left(\begin{array}{l}1 \\ 0\end{array}\right)$

where $|\lambda|<1$

17. $\left\{\mathrm{e}^{t}\left(\begin{array}{ll}1 & t \\ 0 & 1\end{array}\right)\right\} \quad R\left(\begin{array}{l}1 \\ 0\end{array}\right)$

18. $\left\{\left(\begin{array}{ll}a & 0 \\ 0 & b\end{array}\right) \quad \mid a>0, b>0\right\}, R\left(\begin{array}{l}1 \\ 0\end{array}\right)$

19. $\left\{\left(\begin{array}{ll}a & b \\ 0 & a\end{array}\right) \mid a>0\right\}, R\left(\begin{array}{l}1 \\ 0\end{array}\right)$

20. $\left\{\left(\begin{array}{ll}a^{\lambda} & b \\ 0 & a^{\lambda+1}\end{array}\right) \mid a>0\right\}, R\left(\begin{array}{l}1 \\ 0\end{array}\right)$ where $\lambda \in R$

21. $\left\{\left(\begin{array}{ll}a & b \\ 0 & d\end{array}\right) \quad \mid a>0, d>0\right\}, R\left(\begin{array}{l}1 \\ 0\end{array}\right)$

C.

22. $\left\{\left\langle\left(\begin{array}{cc}\mathrm{e}^{t} & 0 \\ 0 & 1\end{array}\right),\left(\begin{array}{l}0 \\ t\end{array}\right)\right\rangle \mid t \in R\right\}$

23. $\left\{\left\langle\left(\begin{array}{ll}1 & t \\ 0 & 1\end{array}\right),\left(\begin{array}{c}t^{2} / 2 \\ t\end{array}\right)\right\rangle \mid t \in R\right\}$

24. $\left\{\left\langle\left(\begin{array}{cc}1 & t \\ 0 & e^{u}\end{array}\right),\left(\begin{array}{l}u \\ 0\end{array}\right)\right\rangle \mid t, u \in R\right\}$

25. $\left\{\left\langle\left(\begin{array}{cc}t^{2} & t u \\ 0 & t\end{array}\right),\left(\begin{array}{c}u^{2} / 2 \\ u\end{array}\right)\right\rangle \mid t>0, u \in R\right\}$

26. $\left\{\left\langle\left(\begin{array}{cc}e^{t} & 0 \\ 0 & 1\end{array}\right),\left(\begin{array}{c}u \\ t\end{array}\right)\right\rangle \mid t, u \in R\right\}$

27. $\left\{\left\langle\left(\begin{array}{cc}1 & t \\ 0 & 1\end{array}\right),\left(\begin{array}{c}u \\ t\end{array}\right)\right\rangle \mid t, u \in R\right\}$

28. $\left\{\left\langle\left(\begin{array}{cc}e^{t} & u \\ 0 & 1\end{array}\right),\left(\begin{array}{c}v \\ t\end{array}\right)\right\rangle \mid t, u, v \in R\right\}$

29. $\left\{\left\langle\left(\begin{array}{cc}t^{2} & t u \\ 0 & t\end{array}\right),\left(\begin{array}{l}v \\ u\end{array}\right)\right\rangle \mid t>0, u, v \in R\right\}$

IV. Generation of subalgebras of $M_{2}(R)$ by pairs of infinitesimal transformations.

Theorem 6. Let $X, Y \in M_{2}(R)$.

a. If $X$ and $Y$ have no common complex eigenvector and $\operatorname{tr} X \neq 0$ or $\operatorname{tr} Y \neq 0, X$ and $Y$ generate $M_{2}(R)$ 
b. If $X$ and $Y$ have no common complex eigenvector and $\operatorname{tr} X=\operatorname{tr} Y$ $=0, X$ and $Y$ generate $s \&(2, R)$

c. If $X$ and $Y$ have a common complex eigenvector and $X, Y,[X, Y]$ are linearly independent, $X$ and $Y$ generate a subalgebra conjugate to $\left\{\left(\begin{array}{ll}a & b \\ 0 & d\end{array}\right)\right\}$

d. Otherwise $X$ and $Y$ generate a subalgebra of dimension $\leqq 2$

e. All subalgebras of $M_{2}(R)$ can be generated by appropriate $X$ and $Y$.

Proof. If $g$ is an algebra listed in Theorem 1 other than $s \ell(2, R)$ and $M_{2}(R)$, there is a complex vector $v$ such that $A v=\lambda v$ for all $A \in g$. Consequently if $X$ and $Y$ have no common complex eigenvector, they generate a subalgebra conjugate to $s \ell(2, R)$ or $M_{2}(R)$. But both of these subalgebras are self-conjugate. The rest of the theorem follows immediately.

REMark. If $g$ is a subalgebra of $M_{2}(R)$ and $X$ and $Y$ belong to $g$, the above theorem gives a satisfactory necessary and sufficient condition that $X$ and $Y$ generate $g$, since the generation problem is trivial when $\operatorname{dim} g \leqq 2$.

V. Generation of subalgebras of $a(2)$ by pairs of infinitesimal transformations. Let $g$ be a subalgebra of $a(2), X$ and $Y$ elements of $g$. We seek a simple necessary and sufficient condition that $X$ and $Y$ generate $g$. The problem is trivial when $\operatorname{dim} g \leqq 2$; if $\operatorname{dim} g=3, X$ and $Y$ generate $g$ just in case $X, Y$, and $[X, Y]$ are linearly independent. When $g \rightarrow g_{0}$ is an isomorphism, the problem was solved in the previous section. Referring to Theorem 4 , we are left with algebras 6 through 12 when $V=R^{2}$ and 21.

TheOREM 7. Let $g=\left\{\langle A, \ell\rangle \mid A \in g_{0}, \ell \in R^{2}\right\}$ where $g_{0}=R\left(\begin{array}{cc}\lambda & -1 \\ 1 & \lambda\end{array}\right)$ $\left.0 \leqq \lambda,\left\{\begin{array}{cc}a & -b \\ b & a\end{array}\right)\right\}$, se $(2, R)$, or $M_{2}(R)$. Let $X=\langle A, l\rangle$ and $Y=\langle B, m\rangle$ belong to $g$. Then $X$ and $Y$ generate $g$ if and only if

(1) A and B generate $g_{0}$

(2) The equations $A v=\ell$ and $B v=m$ cannot be simultaneously solved for $v$; equivalently $A$ is nonsingular and $m \neq B A^{-1} \ell$ or $B$ is nonsingular and $\ell \neq A B^{-1} m$ or $A$ and $B$ are singular and one of $\ell \notin$ range $A, m \notin$ range $B$.

Moreover, such a pair always exists.

Proof. The first condition is obviously necessary. If $v$ satisfies the second condition, conjugation of $X$ and $Y$ by $\langle I, v\rangle$ produces $\langle A, 0\rangle$ and $\langle B, 0\rangle$, so $X$ and $Y$ generate a subalgebra conjugate to $\left\{\langle A, 0\rangle \mid A \in g_{0}\right\}$.

Conversely, suppose 1 and 2 hold; then $X$ and $Y$ generate a sub- 
algebra $\tilde{g}$ such that $\tilde{g}_{0}=g_{0}$. If $\tilde{V}=R^{2}, \tilde{g}=g$; otherwise $\tilde{V}=\{0\}$ by Theorem 3. Then $\tilde{g}$ is conjugate to $\left\{\langle A, 0\rangle \mid A \in g_{0}\right\}$ by Theorem 4 . Let $\langle C, w\rangle \in A(2)$ induce this conjugation; then $X$ and $Y$ become $\left\langle C A C^{-1}, C \ell-C A C^{-1} w\right\rangle$ and $\left\langle C B C^{-1}, C m-C B C^{-1} w\right\rangle$, so $C \ell-$ $C A C^{-1} w=0, C m-C B C^{-1} w=0$; since $C$ is nonsingular, $A\left(C^{-1} w\right)$ $=\ell, B\left(C^{-1} w\right)=m$.

If $A$ is nonsingular, the vector $v$ obviously exists just in case $m=$ $B A^{-1} v$. If $A$ and $B$ generate $g_{0}$ and both are singular, $g_{0}$ must be sl $(2, R)$ or $M_{2}(R)$, so $A$ and $B$ must have rank 1 and $\operatorname{Ker} A \cap \operatorname{Ker} B=$ $\{0\}$. Suppose $\ell \in$ range $A$ and $m \in$ range $B$. Let $A v=\ell$ and suppose that $v_{1}$ generates $\operatorname{Ker} A$; then $A\left(v+\lambda v_{1}\right)=\ell$; since $v_{1} \notin \operatorname{Ker} B, B v_{1}$ generates the range of $B$ and $\lambda$ exists such that $B\left(v+\lambda v_{1}\right)=m$.

The existence of a generating pair is clear.

TheOREM 8. Let $g=\left\{\left\langle\left(\begin{array}{ll}a & 0 \\ 0 & b\end{array}\right), \ell\right\rangle \mid \ell \in R^{2}\right\}$. Let $X=\langle A, \ell\rangle$ and $Y=\langle B, m\rangle$ belong to $g$. Then $X$ and $Y$ generate $g$ if and only if

(1) A and $B$ are linearly independent,

(2) Am - Bl belongs to neither $R_{1}\left(\begin{array}{l}1 \\ 0\end{array}\right)$ nor $R\left(\begin{array}{l}0 \\ 1\end{array}\right)$.

Moreover, $g$ can always be generated by such a pair.

Proof. These conditions are necessary. For example, suppose $A m-B l \in R\left(\begin{array}{l}0 \\ 1\end{array}\right)$. Then the subspace of $g$ generated by $X, Y$, and $[X, Y]=\langle 0, A m-B l\rangle$ is a subalgebra, so $X$ and $Y$ generate an algebra of dimension at most 3 .

Conversely suppose the above conditions hold. Then $X$ and $Y$ generate an algebra $\tilde{g}$ with $\left.\tilde{g}_{0}=\left\{\begin{array}{ll}a & 0 \\ 0 & b\end{array}\right)\right\}$; it is enough to prove that $\tilde{V}=R^{2}$. At any rate $\tilde{V}$ is invariant under $\tilde{g}_{0}$ and so equal to $\{0\}$, $\boldsymbol{R}\left(\begin{array}{l}1 \\ 0\end{array}\right), \boldsymbol{R}\left(\begin{array}{l}0 \\ 1\end{array}\right)$, or $\boldsymbol{R}^{2}$. But $[\langle A, l\rangle,\langle B, m\rangle]=\langle 0, A m-B \ell\rangle$ and $A m-$ $B l \notin R\left(\begin{array}{l}1 \\ 0\end{array}\right)$ or $R\left(\begin{array}{l}0 \\ 1\end{array}\right)$.

The above conditions are satisfied by $X=\left\langle\left(\begin{array}{ll}1 & 0 \\ 0 & 1\end{array}\right), 0\right\rangle$ and $Y=$ $\left\langle\left(\begin{array}{ll}0 & 1 \\ 0 & 1\end{array}\right),\left(\begin{array}{l}1 \\ 1\end{array}\right)\right\rangle$.

TheOREM 9. Let $g=\left\{\langle A, \ell\rangle \mid A \in g_{0}, \ell \in R^{2}\right\}$ where $g_{0}=\left\{\left(\begin{array}{ll}a & b \\ 0 & a\end{array}\right)\right\}$ or $\left\{\left(\begin{array}{ll}a & b \\ 0 & d\end{array}\right)\right\}$. Let $X=\langle A, l\rangle$ and $Y=\langle B, m\rangle$ belong to $g$. Then $X$ and $Y$ generate $g$ if and only if

(1) A and $B$ generate $g_{0}$,

(2) $A m-B l \notin R\left(\begin{array}{l}1 \\ 0\end{array}\right)$.

Moreover, g can always be generated by such a pair.

Proof. These conditions are necessary. Indeed suppose $\mathrm{Am}-$ $B \ell \in R\left(\begin{array}{l}1 \\ 0\end{array}\right)$. If $g_{0}=\left\{\left(\begin{array}{ll}a & b \\ 0 & a\end{array}\right)\right\}$, the subspace generated by $X, Y$, and $\left\langle 0,\left(\begin{array}{l}1 \\ 0\end{array}\right)\right\rangle$ is a subalgebra of dimension at most 3 . If $\left.g_{0}=\left\{\begin{array}{ll}a & b \\ 0 & d\end{array}\right)\right\}$, the subspace generated by $X, Y,\left\langle\left(\begin{array}{ll}0 & 1 \\ 0 & 0\end{array}\right), 0\right\rangle$ and $\left\langle 0,\left(\begin{array}{l}1 \\ 0\end{array}\right)\right\rangle$ is a subalgebra of dimension at most 4 . 
Conversely suppose 1 and 2 hold; then $X$ and $Y$ generate an algebra $\tilde{g}$ with $\tilde{g}_{0}=g_{0}$. It is enough to prove that $\tilde{V}=R^{2}$; at any rate, $\tilde{V}$ is invariant under $g_{0}$ and so $\{0\}, R\left(\begin{array}{l}1 \\ 0\end{array}\right)$, or $R^{2}$. If $g_{0}=\left\{\left(\begin{array}{cc}a & b \\ 0 & a\end{array}\right)\right\}, A m-$ $B \ell \in \tilde{V}$, so $\tilde{V}$ is not $\{0\}$ or $R\left(\begin{array}{l}1 \\ 0\end{array}\right)$. If $g_{0}=\left\{\left(\begin{array}{ll}a & b \\ 0 & d\end{array}\right)\right\rangle$, g contains $\left\langle\left(\begin{array}{ll}1 & 0 \\ 0 & 1\end{array}\right)\right.$, $\left.\left(\begin{array}{l}s_{1} \\ s_{2}\end{array}\right)\right\rangle$ and $[X, Y]=\left\langle\left(\begin{array}{ll}0 & t \\ 0 & 0\end{array}\right),\left(\begin{array}{c}t_{1} \\ t_{2}\end{array}\right)\right\rangle$ for some $s_{1}, s_{2}, t, t_{1}$, and $t_{2}$; we are assuming $t_{2} \neq 0$. Thus $\tilde{g}$ contains the bracket of these two elements, $\left\langle 0,\left(\begin{array}{c}t_{1} \\ t_{2}\end{array}\right)-\left(\begin{array}{c}t_{t_{2}} \\ 0\end{array}\right)\right\rangle$, and $\tilde{V}$ is not $\{0\}$ or $R\left(\begin{array}{l}1 \\ 0\end{array}\right)$.

If $g_{0}=\left\langle\left(\begin{array}{ll}a & b \\ 0 & a\end{array}\right)\right\}$, the conditions are satisfied by $X=\left\langle\left(\begin{array}{ll}1 & 0 \\ 0 & 1\end{array}\right), 0\right\rangle$ and $Y=\left\langle\left(\begin{array}{ll}0 & 1 \\ 0 & 0\end{array}\right),\left(\begin{array}{l}0 \\ 1\end{array}\right)\right\rangle$. If $g_{0}=\left\{\left(\begin{array}{ll}a & b \\ 0 & d\end{array}\right)\right\}$ the conditions are satisfied by $X=\left\langle\left(\begin{array}{ll}0 & 1 \\ 0 & 1\end{array}\right), 0\right\rangle$ and $Y=\left\langle\left(\begin{array}{ll}1 & 0 \\ 0 & 0\end{array}\right),\left(\begin{array}{l}0 \\ 1\end{array}\right)\right\rangle$.

Theorem 10. Let $g=\left\{\left\langle\left(\begin{array}{cc}\lambda a & b \\ 0 & (\lambda+1) a\end{array}\right), \ell\right\rangle \mid a, b \in R, \ell \in R^{2}\right\}$ for $\lambda \in R$. Let $X=\langle A, \ell\rangle$ and $Y=\langle B, m\rangle$ belong to $g$. Then $X$ and $Y$ generate $g$ if and only if

(1) A and $B$ are linearly independent,

(2) $(A-\beta) m-(B+\alpha) l \notin R\left(\begin{array}{l}1 \\ 0\end{array}\right)$ where $[A, B]=\alpha A+\beta B$.

Moreover $g$ can be generated by such a pair unless $\lambda=-2$.

Proof. If $(A-\beta) m-(B-\alpha) \ell \in R\left(\begin{array}{l}1 \\ 0\end{array}\right)$, the subspace generated by $X, Y$, and $\left\langle 0,\left(\begin{array}{l}1 \\ 0\end{array}\right)\right\rangle$ is a subalgebra of dimension at most 3 .

Conversely suppose these conditions hold and let $X$ and $Y$ generate $\tilde{g}$; then $\tilde{g}_{0}=\left\{\left(\begin{array}{cc}\lambda a & b \\ 0 & (\lambda+1) a\end{array}\right)\right\}$ and it is sufficient to show that $\tilde{V}=R^{2}$; since $\tilde{V}$ is invariant under $\tilde{g}_{0}, \tilde{V}=\{0\}, R\left(\frac{1}{0}\right)$, or $R^{2}$. But $\tilde{g}$ contains $[X, Y]-\alpha X-\beta Y=\{0,(A-\beta) m-(B+\alpha) \ell\rangle$, so $\tilde{V}=R^{2}$.

If $\lambda \neq-2$, let $X=\left\langle\left(\begin{array}{cc}\lambda & 0 \\ 0 & \lambda+1\end{array}\right), 0\right\rangle, Y=\left\langle\left(\begin{array}{ll}0 & 1 \\ 0 & 0\end{array}\right),\left(\begin{array}{l}0 \\ 1\end{array}\right)\right\rangle$ and notice that the above conditions hold. If $\lambda=-2$. notice that

$$
\left[\left(\begin{array}{ll}
2 a & b \\
0 & a
\end{array}\right),\left(\begin{array}{ll}
2 c & d \\
0 & c
\end{array}\right)\right]=\left(\begin{array}{ll}
0 & a d-b c \\
0 & 0
\end{array}\right)=-c\left(\begin{array}{ll}
2 a & b \\
0 & a
\end{array}\right)+a\left(\begin{array}{cc}
2 c & d \\
0 & c
\end{array}\right)
$$

and

$$
\begin{gathered}
\left\{\left(\begin{array}{cc}
2 a & b \\
0 & a
\end{array}\right)-a\right\}\left(\begin{array}{l}
m_{x} \\
m_{y}
\end{array}\right)-\left\{\left(\begin{array}{cc}
2 c & d \\
0 & c
\end{array}\right)-c\right\}\left(\begin{array}{l}
\ell_{x} \\
\ell_{y}
\end{array}\right) \\
=\left(\begin{array}{c}
a m_{x}+b m_{y}-c \ell_{x}-d \ell_{y} \\
0
\end{array}\right) .
\end{gathered}
$$

THEOREM 11. Let $g=\left\{\left\langle\left(\begin{array}{ll}a & b \\ 0 & d\end{array}\right),\left(\begin{array}{l}e \\ 0\end{array}\right)\right\rangle\right\}$. Let $X=\langle A, \ell\rangle$ and $Y=$ $\langle B, m\rangle$ belong to $g$. Then $X$ and $Y$ generate $g$ if and only if

(1) A and $B$ generate $\left\{\left(\begin{array}{ll}a & b \\ 0 & d\end{array}\right)\right\}$,

(2) $A m-B l \neq 0$.

Moreover, g can always be generated by such a pair.

Proof. If $A m-B l=0$, the subspace generated by $X, Y$, and $\left\langle\left(\begin{array}{ll}0 & 1 \\ 0 & 0\end{array}\right), 0\right\rangle$ is a subalgebra of dimension at most 3 . 
Conversely suppose these conditions hold and let $X$ and $Y$ generate $\tilde{g}$; then $\left.\tilde{g}_{0}=\left\{\begin{array}{cc}a & b \\ 0 & d\end{array}\right)\right\}$ and it is enough if $\tilde{V} \neq\{0\}$. But $\tilde{g}$ contains $\left\langle\left(\begin{array}{ll}1 & 0 \\ 0 & 1\end{array}\right),\left(\begin{array}{l}s \\ 0\end{array}\right)\right\rangle$ and $[X, Y]=\left\langle\left(\begin{array}{ll}0 & t \\ 0 & 0\end{array}\right),\left(\begin{array}{l}u \\ 0\end{array}\right)\right\rangle$ for some $s, t, u$; we are assuming $u \neq 0$. Thus $\tilde{g}$ contains the bracket of these two elements, $\left\langle 0,\left(\begin{array}{l}u \\ 0\end{array}\right)\right\rangle$, and $\tilde{V} \neq\{0\}$.

The conditions are satisfied by $X=\left\langle\left(\begin{array}{ll}1 & 0 \\ 0 & 0\end{array}\right), 0\right\rangle$ and $Y=\left\langle\left(\begin{array}{ll}0 & 1 \\ 0 & 1\end{array}\right)\right.$, $\left.\left(\begin{array}{l}1 \\ 0\end{array}\right)\right\rangle$.

THEOREM 12. Every connected subgroup of $A(2)$ not conjugate to $\left\{\left\langle\left(\begin{array}{ll}a & 0 \\ 0 & a\end{array}\right), \quad l\right\rangle \mid a>0, \quad \ell \in R^{2}\right\}, \quad\left\{\left\langle\left(\begin{array}{ll}a & b \\ 0 & 1\end{array}\right), \quad\left(\begin{array}{c}c \\ 0\end{array}\right)\right\rangle \mid a>0, b, c \in R\right\}$, or $\left\{\left\langle\left(\begin{array}{cc}a & b \\ 0 & a\end{array}\right), \ell\right\rangle \mid a>0, b \in R, \ell \in R^{2}\right\}$ can be generated by an appropriate pair of infinitesimal transformations.

Proof. It suffices to consider the three dimensional $g$ on the list in Theorem 4. Every non-abelian three dimensional Lie algebra can be generated by appropriate $X$ and $Y$ except the Lie algebra $\left\{\left\langle\left(\begin{array}{ll}a & 0 \\ 0 & a\end{array}\right), \ell\right\rangle\right.$ $\left.\mid a \in R, \ell \in R^{2}\right\}[1]$; we should show that only two algebras on our list are isomorphic to this algebra. It is easier to proceed directly; we already know that 1 through 12 can be generated if $V=\{0\}$. If $V=R^{2}$ pick $X=\left\langle 0,\left(\begin{array}{l}1 \\ 1\end{array}\right)\right\rangle$; let $Y=\left\langle\left(\begin{array}{ll}1 & 0 \\ 0 & \lambda\end{array}\right), 0\right\rangle$ in case 2 if $\lambda \neq 1,\left\langle\left(\begin{array}{cc}\lambda & -1 \\ 1 & \lambda\end{array}\right), 0\right\rangle$ in case 3 if $\lambda \neq 1,\left\langle\left(\begin{array}{ll}0 & 1 \\ 0 & 0\end{array}\right), 0\right\rangle$ in case 4 , and $\left\langle\left(\begin{array}{ll}1 & 1 \\ 0 & 1\end{array}\right), 0\right\rangle$ in case 5 . In case 3 when $\lambda=1$ let $X=\left\langle 0,\left(\begin{array}{l}1 \\ 0\end{array}\right)\right\rangle, Y=\left\langle\left(\begin{array}{cc}1 & -1 \\ 1 & 1\end{array}\right), 0\right\rangle$. In case 18 let $X=\left\langle\left(\begin{array}{ll}1 & 0 \\ 0 & 1\end{array}\right), 0\right\rangle, Y=\left\langle\left(\begin{array}{ll}0 & 0 \\ 0 & 1\end{array}\right),\left(\begin{array}{l}1 \\ 0\end{array}\right)\right\rangle$. In case 19 let $X=\left\langle\left(\begin{array}{ll}1 & 0 \\ 0 & 1\end{array}\right), 0\right\rangle, Y=$ $\left\langle\left(\begin{array}{ll}0 & 1 \\ 0 & 0\end{array}\right),\left(\begin{array}{l}1 \\ 0\end{array}\right)\right\rangle$. In case 20 when $\lambda \neq-1$ let $X=\left\langle\left(\begin{array}{lll}\lambda & 0 \\ 0 & \lambda & 1\end{array}\right), 0\right\rangle, \quad Y=$ $\left\langle\left(\begin{array}{ll}0 & 1 \\ 0 & 0\end{array}\right),\left(\begin{array}{l}1 \\ 0\end{array}\right)\right\rangle$. In case 28 let $\left.X=\left\langle\left(\begin{array}{ll}0 & 1 \\ 0 & 0\end{array}\right), 0\right\rangle, Y=\left\langle\left(\begin{array}{ll}1 & 0 \\ 0 & 0\end{array}\right)\right),\left(\begin{array}{l}0 \\ 1\end{array}\right)\right\rangle$; in case 29 let $X=\left\langle\left(\begin{array}{ll}2 & 0 \\ 0 & 0\end{array}\right), 0\right\rangle, Y=\left\langle\left(\begin{array}{ll}0 & 1 \\ 0 & 0\end{array}\right),\left(\begin{array}{l}1 \\ 1\end{array}\right)\right\rangle$.

Remark. Notice that the only subgroup of $A(2)$ conjugate to $G=$ $\left\{\left\langle\left(\begin{array}{cc}a & 0 \\ 0 & a\end{array}\right), \ell\right\rangle \mid a>0, \ell \in R^{2}\right\}$ is $G$ itself.

\section{REFERENCES}

1. R. Koch and F. Lowenthal, Uniform finite generation of three dimensional linear Lie groups, Can. J. Math. 27 (1975), 396-417.

2. F. Lowenthal, On generating subgroups of the Moebius group by pairs of infinitesimal transformations, Pacific J. Math. 26 (1968), 141-147.

3. - Uniform finite generation of the affine group, Pacific J. Math. 40 (1972), 341-348.

4. Hidehiko Yamabe, On an arcwise connected subgroup of a Lie group, Osaka J. Math. 2 (1950), 13-14.

University of Oregon, Eugene, Oregon

University of Wisconsin at Parkside, Kenosha, Wisconsin 
